\title{
Identification and interpretation of clinical and quality of life prognostic factors for survival and response to treatment in first-line chemotherapy in advanced breast cancer
}

\author{
J.A. Kramer ${ }^{a}$, D. Curran ${ }^{\mathrm{a}, *}$, M. Piccart ${ }^{\mathrm{b}}$, J.C.J.M. de Haes ${ }^{\mathrm{c}}$, P. Bruning ${ }^{\text {d, }}$, \\ J. Klijn ${ }^{\mathrm{e}}$, I. Van Hoorebeeck ${ }^{\mathrm{f}}$, R. Paridaens ${ }^{\mathrm{g}}$ \\ ${ }^{a}$ European Organization for Research and Treatment of Cancer (EORTC) Data Center, Avenue Mounier 83, Bte 11, B-1200, Brussels, Belgium \\ bInstitut Jules Bordet, Brussels, Belgium \\ 'Academic Medical Center, Amsterdam, The Netherlands \\ ${ }^{\mathrm{d}}$ The Netherlands Cancer Institute, Amsterdam, The Netherlands \\ e Daniel den Hoed Cancer Center, Rotterdam, The Netherlands \\ fInvestigational Drug Branch for Breast Cancer, Brussels, Belgium \\ ${ }^{\mathrm{g}}$ University Hospital Gasthuisberg, Leuven, Belgium
}

Received 6 December 1999; received in revised form 22 March 2000; accepted 12 May 2000

\begin{abstract}
The aim of the project was to identify clinical and quality of life (QL) factors that together predict survival and response to chemotherapy in advanced breast cancer. Potential prognostic factors were studied in 187 women with baseline QL data from a trial of paclitaxel versus doxorubicin as first-line chemotherapy. Demographic and clinical factors studied were age, performance status, dominant site of disease and preceding disease-free interval (DFI). Factors from the EORTC QLQ-C30 were all function scales, fatigue, nausea/vomiting, pain, dyspnoea, insomnia, loss of appetite and global QL. The proportional hazards regression model with stratification for treatment, and the logistic regression model adjusting for treatment arm were used for univariate and multivariate analyses of survival and response to treatment, respectively. For survival, multiple sites of visceral disease, pain, global QL and fatigue were significant prognostic factors in the univariate analysis. The final multivariate model predicted poor survival with multiple sites of visceral disease $(P=0.003)$, DFI $\leqslant 2$ years $(P=0.026)$ and pain $(P=0.003)$. For response, age, dyspnoea, fatigue and global QL were significant predictive factors in the univariate analysis. The final multivariate model for response selected DFI $(P=0.009)$, multiple sites of visceral disease $(P=0.037)$ and dyspnoea $(P=<0.001)$ using forward selection, but model instability was indicated by the inclusion of fatigue and emotional function in the final model when backward selection was used. In addition to known clinical factors, patient-assessed QL variables appear to be prognostic for survival and response to chemotherapy in women with advanced breast cancer. However, identification of prognostic factors from responses to questionnaires may be unstable, and their reliability and clinical utility should be tested prospectively. (C) 2000 Elsevier Science Ltd. All rights reserved.
\end{abstract}

Keywords: Prognostic factor; Breast cancer; Quality of life; EORTC QLQ-C30

\section{Introduction}

Prognostic factor analyses are used to identify variables that should be used to stratify patients in the design and analysis of clinical trials, to assist in the interpretation of the data generated by such trials, and

* Corresponding author. Tel.: + 353-1-2161-284; fax +353-1-2161200.

E-mail address: currand@iconirl.com (D. Curran). to aid in the clinical management of individual cancer patients. Many demographic and clinical variables have been identified as prognostic factors for women with advanced breast cancer, including age, menopausal status, hormone receptor status, performance status, sites of metastases, prior adjuvant chemotherapy and disease-free interval (DFI). Whilst identification of factors that are prognostic for survival or predictive of response to treatment is intended to assist clinicians, such information is often difficult to use in practice because of conflicting results arising from differences in the design 
and methodology of clinical trials [1] and in the statistical analyses employed [2].

Recently, several exploratory studies have suggested that quality of life (QL) and other patient-assessed variables have 'independent' prognostic significance, raising the possibility that they add important prognostic information to that provided by clinical variables. This finding is potentially important for the design of studies, particularly those involving palliative therapies. Studies using linear analogue self-assessment (LASA) scales [3-5], the Functional Living Index in Cancer (FLIC) [6,7], the Therapy Impact Questionnaire (TIQ) $[8,9]$, the Rotterdam Symptom Checklist $[10,11]$ and other instruments $[12,13]$ have suggested that many QL variables might have prognostic importance.

The European Organization for Research and Treatment of Cancer (EORTC) QLQ-C30 quality of life core questionnaire [14] has also been used to identify prognostic factors. Coates and colleagues [15] and Dancey and associates [16] studied large heterogeneous groups of patients with cancer and entered baseline QL variables measured using the EORTC QLQ-C30 into multivariate models containing clinical prognostic factors such as performance status. Good social function and global QL were associated with a longer survival in advanced cancer [15]. A good global QL score was associated with a better prognosis in a mixed group of cancer patients about to receive their first course of chemotherapy, but poor emotional function (score below the mean for the group) also predicted longer survival [16]. Tannock and associates [17] studied patients with hormone-resistant prostate cancer, and found that low pain intensity and good physical function at baseline were associated with a better prognosis.

Part of the difficulty of interpreting such studies arises from the self-assessment questionnaires themselves, in which individual items or subscales may be highly correlated. The literature suggests that the results of prognostic factor analyses using variables derived from questionnaires could be unstable because of high correlation between variables. Coates and colleagues [5] noted considerable correlation between variables measured using LASA scales, and observed that minor variations in their correlations may dictate which variables remain in the final regression model that determines prognostic factors. Strong correlations have also been observed between items on the QLQ-C30 [14]. In multiple regression when some or all of the variables are highly correlated, it is difficult to disentangle their influences and obtain an estimate of their individual effects [18]. The statistical handling of this phenomenon, known as multicollinearity, is complex and will be the subject of a companion paper investigating its impact on the interpretation of prognostic factor analyses using patient-assessed variables.
We report here an exploratory prognostic factor analysis for response and survival of clinical and QL data from a randomised phase II/III trial comparing paclitaxel and doxorubicin as first-line therapy in women with advanced breast cancer. No substantial difference in survival or response was expected between the treatments, and so measurement of QL was considered particularly important in this trial. We also considered it important to study the correlations between items measured using the QLQ-C30 in order to observe their influence in determining the variables selected for final multivariate models.

\section{Patients and methods}

EORTC trial 10923 was a prospective, randomised phase II/III crossover study in advanced breast cancer, in which the efficacy of paclitaxel $200 \mathrm{mg} / \mathrm{m}^{2}$ as a 3-h infusion every 3 weeks until progression followed by doxorubicin $75 \mathrm{mg} / \mathrm{m}^{2}$ (maximum seven courses) as an intravenous (i.v.) bolus every 3 weeks, was compared with the reverse regimen of doxorubicin followed by paclitaxel. The trial was approved by the EORTC Protocol Review Committee and by the ethics committee of each participating centre, and was conducted in compliance with the Helsinki declaration. All patients gave informed consent.

\subsection{Patients}

To be eligible, patients were required to be female with histologically or cytologically proven incurable adenocarcinoma of the breast in overt progression, with uni- or bidimensionally measurable lesion(s) not previously treated with chemotherapy for advanced disease. Patients that were pregnant or lactating were ineligible. Other requirements for eligibility were Eastern Cooperative Oncology Group (ECOG)/World Health Organization (WHO) performance status (PS) $\leqslant 2$; age $\geqslant 18$ years; life expectancy $\geqslant 3$ months; and adequate haematological, renal, hepatic and cardiac function. Patients were ineligible if they had a past or current history of any neoplasm other than breast carcinoma; relapse from previous adjuvant chemotherapy within 3 months of its completion or adjuvant chemotherapy containing anthracyclines; active serious infection; or the presence of symptomatic central nervous system (CNS) metastases.

\subsection{Clinical methods}

Baseline clinical data included age, performance status, dominant site of disease, details of prior therapy and date of first relapse. Dominant site of disease was defined according to International Union Against Cancer 
(UICC) criteria [19]. The EORTC quality of life core questionnaire (QLQ-C30 v. 1.0) was used to assess QL [14]. The schedule of QL assessments required a baseline measurement at randomisation $(\leqslant 2$ weeks before starting treatment), further assessments at the end of the third, fifth and seventh cycles of chemotherapy for both first and second-line treatments, and when the patient went off study for any reason except death. For the purpose of this report, only baseline questionnaires were considered.

When the study was designed the Rotterdam Symptom Checklist (RSCL) [20] was already well established, whereas the EORTC QLQ-C30 had only recently been validated. Therefore, both instruments were used to assess QL to try to ensure that any true differences in QL between the treatments were detected. Only the QLQ-C30 was used for the prognostic factor analysis reported here, in order to limit the number of variables studied. The QLQ-C30 consists of five function scales: physical (PF), role (RF), emotional (EF), cognitive (CF) and social (SF); three symptom scales: fatigue (FA), nausea/vomiting (NV) and pain (PA); six single-item scales (dyspnoea (DY), sleep disturbance (SL), appetite loss (AP), constipation (CO), diarrhoea (DI) and financial impact of the disease/treatment (FI)), and a global health status/quality of life (QL) scale. Items were scored and scales constructed using the recommended procedures [21]. In order to limit the number of variables under consideration, three single-item scales (CO, DI and FI) were not included in the prognostic factor analysis.

Survival times were measured from the date of randomisation. Survival times of patients who were known to be alive at the date of last follow-up were censored at that date.

Tumour response was assessed according to UICC criteria [19]. Complete remission was defined as the disappearance of all known disease, determined by two observations not less than 4 weeks apart. Partial response was defined as a decrease of at least $50 \%$ in the sum of the products of the largest perpendicular diameters of all measurable lesions (determined by two observations not less than 4 weeks apart) in the case of bidimensionally measurable disease, or a decrease of at least $50 \%$ of the sum of the largest diameters of all lesions for unidimensionally measurable disease. It was not necessary for all lesions to have regressed to qualify for a partial response, but no lesion should have progressed and no new lesion should have appeared.

\subsection{Variables studied}

The following factors were studied: age, performance status (PS), dominant site of disease, disease-free interval and QLQ-C30 variables. To minimise bias in selection, variables were dichotomised at the median where possible. PS was dichotomised at score 0 versus scores 1 and 2 combined, because only $8 \%$ patients had PS $=2$ at baseline. Disease-free interval (DFI) was grouped into newly diagnosed patients with advanced disease $(\mathrm{DFI}=0), \mathrm{DFI} \leqslant 2$ years and DFI $>2$ years.

For variables measured using the QLQ-C30, raw scores were transformed to a linear scale ranging from 0 to 100 , with higher scores representing a higher level of functioning or a higher level of symptoms [21]. In the case of missing items within a scale, the scale score was calculated using only those for which values were available, provided at least half of the items in the scale were completed. All QL variables except cognitive function (CF) were subsequently dichotomised at the median to yield 'good' and 'poor' scores. CF was dichotomised at a score of 70 because the median score was 83 , and all scores at or below this value cannot necessarily be classed as 'poor' cognitive function on a scale of zero to 100.

\subsection{Statistical considerations}

Randomisation was performed using the minimisation technique [22]. The primary endpoint of the trial was progression-free survival. QL, response to treatment and overall survival were secondary endpoints.

Survival curves and probabilities were estimated using the Kaplan-Meier technique [23]. The Cox proportional hazards regression model with stratification for treatment arm was used for both univariate and multivariate analyses of survival. The logistic regression model with treatment arm included in the model was used for both univariate and multivariate analyses of response.

Clinical factors were entered first to build the final clinical models for survival and response, and QLQ-C30 variables were then added. A step-up (forward) variable selection procedure was used to identify QL variables that contributed additional prognostic information to the multivariate models for survival and response. For comparative purposes multivariate models using stepdown (backward) elimination procedures were also fitted.

For the survival prognostic factor analysis the proportionality assumption was checked for each of the variables under study by testing the dependency of their hazard ratio over time [24]. The importance of a prognostic factor was assessed using the $P$ value of the Wald $\chi^{2}$ statistic, the hazard ratio and the odds ratio (risk in a given category compared with the reference one) and its $95 \%$ confidence interval for survival and response, respectively.

Spearman rank correlation $\left(\mathrm{r}_{\mathrm{s}}\right)$ was used to investigate relationships between QLQ-C30 variables. Data analysis was performed using Statistical Analysis Software (SAS) [25]. A 5\% level of significance was used for clinical variables, and a $1 \%$ level of significance was chosen for patient-assessed variables to reduce the risk 
of false-positive results arising from multiple testing. All significance tests were two-sided.

\section{Results}

Between September 1993 and April 1996, 331 patients from 20 institutions were randomised into the study. 15 patients were ineligible on clinical grounds. A further 22 patients were not eligible for the QL analysis. 187 of 294 eligible patients completed baseline QL evaluations, giving a compliance rate of $64 \%$. Further details are provided in our companion paper in this issue of the European Journal of Cancer (pp. 1488-1497).

Characteristics of all eligible patients in the trial are reported elsewhere [26]. Characteristics of 187 eligible patients with baseline QLQ-C30 questionnaires are shown in Table 1. Univariate analyses were performed on data from these 187 patients, but 10 without complete information on all QL variables were excluded from the multivariate analyses, leaving 177 patients for whom complete information was available on all variables for these analyses. For the clinical prognostic factors of age, performance status, disease-free interval and dominant site of disease, no significant difference in the distribution of these factors was detected between eligible patients included and those excluded from the QL analysis.

Table 1

Characteristics of eligible patients with baseline QL questionnaires $(n=187)$

\begin{tabular}{lcc}
\hline & Paclitaxel $n(\%)$ & Doxorubicin $n(\%)$ \\
\hline Age (years) & & \\
$\quad>55$ & $44(47)$ & $41(44)$ \\
$\leqslant 55$ & $50(53)$ & $52(56)$ \\
WHO performance status & & \\
0 & $40(43)$ & $36(39)$ \\
1 & $45(48)$ & $51(55)$ \\
2 & $9(10)$ & $6(6)$ \\
Disease-free interval & & \\
$=0$ (Newly diagnosed) & $8(9)$ & $8(9)$ \\
$\leqslant 2$ years & $40(43)$ & $28(30)$ \\
$>2$ years & $46(49)$ & $57(61)$ \\
Prior therapy & & \\
Surgery & & \\
$\quad$ Mastectomy & $37(39)$ & $47(51)$ \\
$\quad$ Breast conserving & $49(52)$ & $39(42)$ \\
$\quad$ Other (including none) & $8(9)$ & $7(8)$ \\
Radiotherapy & $78(83)$ & $73(78)$ \\
Adjuvant therapy (C, H, I) & $44(47)$ & $57(61)$ \\
H, I for advanced disease & $55(59)$ & $57(61)$ \\
Dominant site of disease & & \\
Soft tissue only & $4(4)$ & $9(10)$ \\
Bone \pm soft tissue & $14(15)$ & $12(13)$ \\
Single visceral & $62(66)$ & $56(60)$ \\
Multiple visceral & $14(15)$ & $16(17)$ \\
\hline
\end{tabular}

C, chemotherapy; H, hormone therapy; I, immunotherapy.
The median interval between initial diagnosis and randomisation in the trial was 4.1 years. The median interval between first relapse and randomisation was 7 months. At the time of analysis, 154/187 (82\%) deaths had been reported, with a median survival of 1.4 years. Median follow-up was 3.6 years. In the paclitaxel arm there were $80 / 94(85 \%)$ deaths and in the doxorubicin arm $74 / 93(80 \%)$ for a median survival of 1.3 and 1.5 years, respectively. Response rates were 23/94 (24\%) for paclitaxel and 38/93 (41\%) for doxorubicin.

\subsection{Prognostic factors for survival}

\subsubsection{Univariate analysis for survival}

Table 2 lists the results of the univariate survival analysis for each of the variables under study. Poor survival was associated with multiple sites of visceral disease, pain, poor global QL and fatigue at baseline, with poor physical function having borderline significance. No other variables were significantly predictive of survival duration. No major violations of the proportionality assumptions were detected for any of the variables studied.

\subsubsection{Multivariate analysis for survival}

The Cox multivariate model, stratifying by treatment arm, retained two clinical prognostic factors: multiple sites of visceral disease $(P=0.001)$ and DFI $\leqslant 2$ years $P=0.007)$. The final model, with QLQ-C30 variables added to the two significant clinical variables, indicated that poor survival was associated with multiple sites of visceral disease $(P=0.003)$, DFI $\leqslant 2$ years $(P=0.026)$ and pain $(P=0.003)$. The backward elimination method produced the same result. Fig. 1 shows survival according to the baseline pain score.
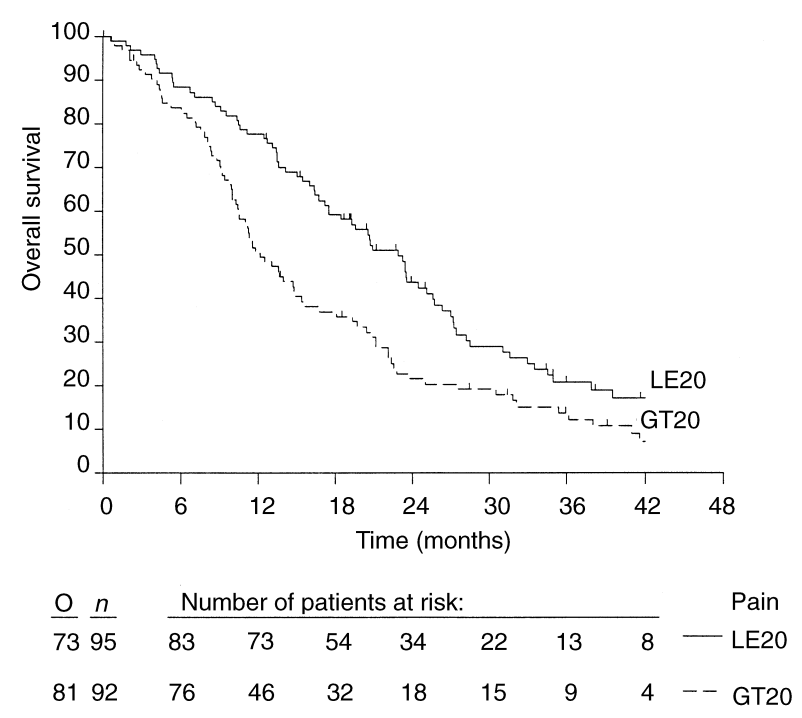

Fig. 1. Duration of survival by QLQ-C30 pain score $(\leqslant 20$ versus $>20$ ). O, observed number of deaths; $n$, number of patients; LE20, $\leqslant 20$; GT20, $>20$. 
Table 2

Univariate prognostic factor analysis for survival ${ }^{\mathrm{a}}$

\begin{tabular}{|c|c|c|c|c|}
\hline Variable & $\mathrm{O} / n(\%)$ & Median survival (years) & Hazard ratio ( $95 \%$ confidence interval) & $P$ value \\
\hline \multicolumn{5}{|l|}{ WHO PS } \\
\hline $0^{\mathrm{b}}$ & $62 / 76(82)$ & 1.6 & 1.0 & \\
\hline 1,2 & $92 / 111(83)$ & 1.3 & $1.08(0.78-1.50)$ & 0.648 \\
\hline \multicolumn{5}{|l|}{ Age } \\
\hline$>55$ years $^{\mathrm{b}}$ & $70 / 85(82)$ & 1.3 & 1.0 & \\
\hline$\leqslant 55$ years & $84 / 102(82)$ & 1.5 & $1.0(0.72-1.38)$ & 0.989 \\
\hline \multicolumn{5}{|l|}{ Dominant site } \\
\hline Soft tissue $\mathrm{b}^{\mathrm{b}}$ & $9 / 13(69)$ & 1.9 & 1.0 & \\
\hline Bone & $22 / 26(85)$ & 1.7 & $1.39(0.63-3.07)$ & 0.416 \\
\hline Single visceral & $96 / 118(81)$ & 1.6 & $1.45(0.73-2.90)$ & 0.291 \\
\hline Multiple visceral & $27 / 30(90)$ & 1.1 & $2.60(1.21-5.59)$ & 0.014 \\
\hline \multicolumn{5}{|l|}{ Disease-free interval } \\
\hline$=0^{\mathrm{b}}$ & $14 / 16(88)$ & 1.9 & 1.0 & \\
\hline$\leqslant 2$ years & $59 / 68(87)$ & 1.1 & $1.38(0.77-2.48)$ & 0.279 \\
\hline$>2$ years & $81 / 103(79)$ & 1.7 & $0.92(0.52-1.63)$ & 0.778 \\
\hline \multicolumn{5}{|l|}{ Physical function } \\
\hline$>60^{\mathrm{b}}$ & 70/89 (79) & 1.8 & 1.0 & \\
\hline$\leqslant 60$ & $84 / 98(86)$ & 1.1 & $1.52(1.10-2.10)$ & 0.010 \\
\hline \multicolumn{5}{|l|}{ Role function } \\
\hline$>50^{\mathrm{b}}$ & $68 / 84(81)$ & 1.7 & 1.0 & \\
\hline$\leqslant 50$ & $86 / 103(83)$ & 1.1 & $1.42(1.02-1.96)$ & 0.037 \\
\hline \multicolumn{5}{|l|}{ Emotional function } \\
\hline$>60^{\mathrm{b}}$ & 89/108 (82) & 1.5 & 1.0 & \\
\hline$\leqslant 60$ & $63 / 76(83)$ & 1.2 & $1.02(0.74-1.42)$ & 0.892 \\
\hline \multicolumn{5}{|l|}{ Cognitive function } \\
\hline$>70^{\mathrm{b}}$ & 108/130 (83) & 1.4 & 1.0 & \\
\hline$\leqslant 70$ & $44 / 54(81)$ & 1.4 & $0.87(0.61-1.25)$ & 0.443 \\
\hline \multicolumn{5}{|l|}{ Social function } \\
\hline$>70^{\mathrm{b}}$ & 89/108 (82) & 1.5 & 1.0 & \\
\hline$\leqslant 70$ & $63 / 76(83)$ & 1.1 & $1.20(0.87-1.67)$ & 0.272 \\
\hline \multicolumn{5}{|l|}{ Global quality of life } \\
\hline$>60^{\mathrm{b}}$ & 73/94 (78) & 1.7 & 1.0 & \\
\hline$\leqslant 60$ & $76 / 87(87)$ & 0.9 & $1.61(1.17-2.24)$ & 0.004 \\
\hline \multicolumn{5}{|l|}{ Fatigue } \\
\hline$\leqslant 40^{\mathrm{b}}$ & $88 / 110(80)$ & 1.7 & 1.0 & \\
\hline$>40$ & $65 / 76(86)$ & 1.0 & $1.55(1.12-2.14)$ & 0.009 \\
\hline \multicolumn{5}{|l|}{ Nausea/vomiting } \\
\hline$=0^{\mathrm{b}}$ & $108 / 130(83)$ & 1.6 & 1.0 & \\
\hline$>0$ & $46 / 57(81)$ & 1.0 & $1.26(0.89-1.79)$ & 0.202 \\
\hline \multicolumn{5}{|l|}{ Pain } \\
\hline$\leqslant 20^{\mathrm{b}}$ & 73/95 (77) & 1.9 & 1.0 & \\
\hline$>20$ & $81 / 92(88)$ & 1.0 & $1.64(1.19-2.27)$ & 0.003 \\
\hline \multicolumn{5}{|l|}{ Dyspnoea } \\
\hline$=0^{\mathrm{b}}$ & $82 / 100(82)$ & 1.7 & 1.0 & \\
\hline$>0$ & $70 / 83(84)$ & 1.1 & $1.35(0.98-1.87)$ & 0.070 \\
\hline \multicolumn{5}{|l|}{ Insomnia } \\
\hline$=0^{\mathrm{b}}$ & $58 / 70(83)$ & 1.3 & 1.0 & \\
\hline$>0$ & $95 / 115(83)$ & 1.6 & $0.91(0.65-1.27)$ & 0.575 \\
\hline \multicolumn{5}{|l|}{ Loss of appetite } \\
\hline$=0^{\mathrm{b}}$ & 76/95 (80) & 1.6 & 1.0 & \\
\hline$>0$ & 78/92 (85) & 1.1 & $1.32(0.95-1.82)$ & 0.095 \\
\hline
\end{tabular}

$\mathrm{O}$, observed number of deaths; $n$, number of patients.

a 10 patients had missing scores for at least one item.

b Reference category. 
Table 3

Univariate prognostic factor analysis for response $\mathrm{e}^{\mathrm{a}}$

\begin{tabular}{|c|c|c|c|}
\hline Variable & $\mathrm{R} / n(\%)$ & Odds ratio ( $95 \%$ confidence interval) & $P$ value \\
\hline \multicolumn{4}{|l|}{ WHO PS } \\
\hline $0^{\mathrm{b}}$ & $27 / 76(36)$ & 1 & \\
\hline 1,2 & $34 / 111(31)$ & $0.77(0.41-1.45)$ & 0.419 \\
\hline \multicolumn{4}{|l|}{ Age } \\
\hline$>55$ years $^{\mathrm{b}}$ & $34 / 85(40)$ & 1 & \\
\hline$\leqslant 55$ years & $27 / 102(26)$ & $0.52(0.28-0.97)$ & 0.040 \\
\hline \multicolumn{4}{|l|}{ Dominant site } \\
\hline Soft tissue ${ }^{b}$ & $5 / 13(38)$ & 1 & \\
\hline Bone & $8 / 26(31)$ & $0.85(0.20-3.50)$ & 0.817 \\
\hline Single visceral & $43 / 118(36)$ & $1.09(0.33-3.63)$ & 0.890 \\
\hline Multiple visceral & $5 / 30(17)$ & $0.35(0.08-1.56)$ & 0.170 \\
\hline \multicolumn{4}{|l|}{ Disease-free interval } \\
\hline$=0^{\mathrm{b}}$ & $8 / 16(50)$ & 1 & \\
\hline$\leqslant 2$ years & $14 / 68(21)$ & $0.27(0.08-0.85)$ & 0.025 \\
\hline$>2$ years & $39 / 103(38)$ & $0.58(0.20-1.70)$ & 0.318 \\
\hline \multicolumn{4}{|l|}{ Physical function } \\
\hline$>60^{\mathrm{b}}$ & $37 / 89(42)$ & 1 & \\
\hline$\leqslant 60$ & $24 / 98(24)$ & $0.46(0.24-0.86)$ & 0.016 \\
\hline \multicolumn{4}{|l|}{ Role function } \\
\hline$>50^{\mathrm{b}}$ & $32 / 84(38)$ & 1 & \\
\hline$\leqslant 50$ & 29/103 (28) & $0.59(0.31-1.10)$ & 0.097 \\
\hline \multicolumn{4}{|l|}{ Emotional function } \\
\hline$>60^{\mathrm{b}}$ & $32 / 108(30)$ & 1 & \\
\hline$\leqslant 60$ & 28/76 (37) & $1.35(0.72-2.55)$ & 0.346 \\
\hline \multicolumn{4}{|l|}{ Cognitive function } \\
\hline$>70^{\mathrm{b}}$ & $46 / 130(35)$ & 1 & \\
\hline$\leqslant 70$ & $14 / 54(26)$ & $0.63(0.31-1.29)$ & 0.209 \\
\hline \multicolumn{4}{|l|}{ Social function } \\
\hline$>70^{\mathrm{b}}$ & 41/108 (38) & 1 & \\
\hline$\leqslant 70$ & $19 / 76(25)$ & $0.51(0.26-0.98)$ & 0.045 \\
\hline \multicolumn{4}{|l|}{ Global quality of life } \\
\hline$>60^{\mathrm{b}}$ & $39 / 94(41)$ & 1 & \\
\hline$\leqslant 60$ & 19/87 (22) & $0.40(0.21-0.78)$ & 0.007 \\
\hline \multicolumn{4}{|l|}{ Fatigue } \\
\hline$\leqslant 40^{\mathrm{b}}$ & $44 / 110(40)$ & 1 & \\
\hline$>40$ & 16/76 (21) & $0.36(0.18-0.72)$ & 0.004 \\
\hline \multicolumn{4}{|l|}{ Nausea/vomiting } \\
\hline$=0^{\mathrm{b}}$ & 49/130 (38) & 1 & \\
\hline$>0$ & $12 / 57(21)$ & $0.41(0.19-0.85)$ & 0.018 \\
\hline \multicolumn{4}{|l|}{ Pain } \\
\hline$\leqslant 20^{\mathrm{b}}$ & $34 / 95(36)$ & 1 & \\
\hline$>20$ & 27/92 (29) & $0.76(0.41-1.42)$ & 0.388 \\
\hline \multicolumn{4}{|l|}{ Dyspnoea } \\
\hline$=0^{\mathrm{b}}$ & $42 / 100(42)$ & 1 & \\
\hline$>0$ & 17/83 (20) & $0.35(0.18-0.68)$ & 0.002 \\
\hline \multicolumn{4}{|l|}{ Insomnia } \\
\hline$=0^{\mathrm{b}}$ & $23 / 70(33)$ & 1 & \\
\hline$>0$ & $37 / 115(32)$ & $1.01(0.53-1.91)$ & 0.989 \\
\hline \multicolumn{4}{|l|}{ Loss of appetite } \\
\hline$=0^{\mathrm{b}}$ & $38 / 95(40)$ & 1 & \\
\hline$>0$ & $23 / 92(25)$ & $0.49(0.26-0.92)$ & 0.027 \\
\hline
\end{tabular}

$\mathrm{R}$, observed number of responses; $n$, number of patients.

a 10 patients had missing scores for at least one item.

b Reference category. 


\subsection{Prognostic factors for response}

\subsubsection{Univariate analysis for response}

Poor response was associated with age, DFI $\leqslant 2$ years, dyspnoea, fatigue and poor global QL. Poor physical function and nausea/vomiting at baseline had borderline significance (Table 3). No other variables were significantly predictive of response.

\subsubsection{Multivariate analysis for response}

The logistic regression model for response with treatment arm included in the model retained the same clinical variables as the multivariate survival analysis: multiple sites of visceral disease $(P=0.028)$ and DFI $\leqslant 2$ years $(P=0.013)$. The final model including QLQ-C30 variables predicted a poor response with multiple sites of visceral disease $(P=0.037)$, DFI $(P=0.009)$ and dyspnoea $(P<0.001)$. The backward elimination method included FA and EF in the model: DFI $\leqslant 2$ years $(P=0.004)$, multiple sites of visceral disease $(P=0.028)$, dyspnoea $(P=0.003)$, good EF $(P=0.008)$ and fatigue $(P=0.004)$.

\subsection{Correlation matrix for $Q L Q-C 30$ variables}

The variables most strongly associated with pain were physical, role, social functioning, global health status/ QL $\left(r_{\mathrm{s}}<-0.4\right)$ and fatigue $\left(\mathrm{r}_{\mathrm{s}}>0.4\right)$; with dyspnoea they were global health status/QL $\left(r_{s}<-0.4\right)$ and fatigue $\left(r_{s}>0.4\right)$ (Table 4). Multicollinearity between items and scales was evident and many variables were strongly intercorrelated $\left(\left|\mathrm{r}_{\mathrm{s}}\right|>0.4\right)$. Variables with the lowest correlations were EF, SL, CF, DY and NV.

\section{Discussion}

This study has shown that QLQ-C30 variables are significant predictors of survival and response to treatment in advanced breast cancer in addition to clinical variables. Patient characteristics of those with QL data available suggest some differences between treatment arms but these do not affect the analysis since the survival model was stratified by treatment arm and the response model was adjusted for treatment effect. The significant clinical variables retained in multivariate models were DFI $\leqslant 2$ years and dominant site of disease. Patients who relapsed within 2 years of diagnosis may have had more rapidly progressive disease, and multiple sites of visceral disease are already known to be associated with a poor outcome. Neither age nor performance status (PS) were retained in the final multivariate models for response and survival, although both are frequently reported to have prognostic importance and age was just significant for response in our univariate analysis. Our negative finding for PS might be partly an effect of sample size of the QL data set, as WHO PS was shown to have borderline significance as a prognostic factor in the analysis of the full dataset. Furthermore, eligibility for this study was restricted to patients with favourable PS, and only $8 \%$ had $\mathrm{PS}=2$, limiting the influence of this variable as a prognostic factor.

Oestrogen and progesterone receptor (ER and PR) status of the primary tumour have previously been identified as significant prognostic factors for survival in advanced breast cancer [27-29]. However, Blanco and associates demonstrated that these variables were not retained in a multivariate model which included DFI, dominant site of disease and histological grading [29]. The authors concluded that ER status and site of recurrence were correlated with high concentrations of ER found in patients with bone metastases, whereas lower concentrations of ER were related to visceral recurrence. Information on ER and PR status, and menopausal status at initial diagnosis were collected in this study. However, the proportion of patients with missing data for ER and PR status was $30 \%$ and $37 \%$, respectively. Because of the high proportion of missing data and owing to the fact that these variables were reflecting the status of patients at initial diagnosis (note: the median interval between initial diagnosis and

Table 4

Correlation matrix for EORTC QLQ-C30 function variables, symptoms and global quality of life (Spearman correlation coefficients, $n=177$ )

\begin{tabular}{|c|c|c|c|c|c|c|c|c|c|c|c|}
\hline & $\mathrm{PF}$ & RF & $\mathrm{EF}$ & $\mathrm{CF}$ & $\mathrm{SF}$ & FA & NV & PA & DY & $\mathrm{SL}$ & AP \\
\hline Role Function (RF) & 0.706 & & & & & & & & & & \\
\hline Emotional Function (EF) & 0.151 & 0.196 & & & & & & & & & \\
\hline Cognitive Function (CF) & 0.268 & 0.236 & 0.361 & & & & & & & & \\
\hline Social Function (SF) & 0.496 & 0.549 & 0.322 & 0.408 & & & & & & & \\
\hline Fatigue (FA) & -0.657 & -0.573 & -0.359 & -0.464 & -0.584 & & & & & & \\
\hline Nausea/Vomiting (NV) & -0.353 & -0.339 & -0.110 & -0.151 & -0.308 & 0.421 & & & & & \\
\hline Pain (PA) & -0.495 & -0.457 & -0.189 & -0.294 & -0.453 & 0.511 & 0.315 & & & & \\
\hline Dyspnoea (DY) & -0.375 & -0.303 & -0.288 & -0.246 & -0.345 & 0.489 & 0.294 & 0.142 & & & \\
\hline Insomnia (SL) & -0.044 & -0.201 & -0.301 & -0.116 & -0.186 & 0.203 & 0.117 & 0.201 & -0.037 & & \\
\hline Appetite loss (AP) & -0.374 & -0.344 & -0.208 & -0.356 & -0.361 & 0.505 & 0.489 & 0.318 & 0.324 & 0.104 & \\
\hline Global health status $\backslash \mathrm{QL}$ & 0.578 & 0.523 & 0.332 & 0.319 & 0.674 & -0.709 & -0.363 & -0.535 & -0.422 & -0.253 & -0.413 \\
\hline
\end{tabular}


randomisation into the trial was 4.1 years) these variables were not considered in the analysis.

For survival, the univariate analysis showed that multiple sites of visceral disease, pain, poor global QL and fatigue were strongly predictive of a poor prognosis, while poor physical function had a borderline significance. For poor response to treatment, the significant univariate predictors were age, DFI $\leqslant 2$ yrs dyspnoea, fatigue and poor global QL, with poor physical function, as well as nausea/vomiting, again having borderline significance. Fatigue, poor global QL and poor physical function are manifestations of faradvanced disease, so that women with poor scores for these variables may have been near death and less likely to respond to treatment. Thus, the important differences between the two models were pain for survival, and dyspnoea for response to treatment.

Pain has been reported to have prognostic significance in symptomatic hormone-resistant prostate cancer [17] and after adjusting for clinical factors in advanced nonsmall cell lung cancer [30]. The results of the present study suggest that pain also has prognostic importance for survival in advanced breast cancer. This is an important finding, because pain is measurable, potentially allowing clinicians to distinguish those patients for whom chemotherapy might be more of a burden than beneficial in future studies. However, just because pain appears to be an indicator of poor prognosis does not imply chemotherapy will benefit these patients. Moreover, pain is also amenable to palliative treatment with radiotherapy, analgesics and a variety of adjuvant measures, thus allowing patients a number of treatment options apart from chemotherapy.

Dyspnoea, a symptom that can result directly from lung pathology, cardiac disease or general manifestations of chronic systemic disease such as anaemia, appears to be important for predicting response to treatment in advanced breast cancer. Ramirez and associates [31] used the RSCL to identify pretreatment QL variables that predicted feeling worse or failing treatment in a consecutive series of women receiving chemotherapy for advanced breast cancer and found that lacking energy and dyspnoea, together with liver metastases predicted treatment failure. This is consistent with our findings that dyspnoea and multiple sites of visceral disease are associated with poor response to chemotherapy.

In this study, different selection methods for multivariate analysis produced different results for response, with backward elimination including fatigue and good emotional function with dyspnoea as predictors of poor response. It is difficult to produce a plausible interpretation for the apparent inverse association between emotional function and response, but we are not the first to have observed this phenomenon using the QLQC30. Good emotional function has previously been associated with shorter survival in a general population of cancer patients receiving chemotherapy [16]. The consistent findings of these two studies suggest that this phenomenon warrants further study.

Instability in the model predicting response to treatment arises from the multicollinearity inherently present between subscales of questionnaires. The influences determining the selection of variables in multivariate prognostic factor analyses based on QL questionnaires have not been investigated systematically and are the subject of a companion paper in preparation. However, the correlation matrix and the instability of model selection for response point to the need for caution when reporting prognostic factors in this context. Reports of multivariate prognostic factor analyses commonly state that statistically significant variables retained in the model are 'independently predictive' of the outcome of interest (response or survival). This terminology is clearly inaccurate in the case of variables originating from questionnaires especially when models select global QL as a significant prognostic factor [15]. The correlation matrix (Table 4) shows that no variable can be regarded as an 'independent predictor' of global QL since they are all interrelated. It is not surprising, therefore, that small differences in their correlations may dictate which variables remain in the final regression model. Hauck and Miike [32] have drawn attention to the danger of overinterpreting the selected variables as the most important ones in procedures on correlated sets of variables, and we have observed that variables from questionnaires are particularly prone to this difficulty. Our findings support the need identified by Simon and Altman [2] for prospective confirmatory studies with specific hypotheses and with particular attention paid to limiting and controlling problems of multiplicity. We chose levels of 0.01 instead of the usual 0.05 to define the statistical significance of patient-assessed variables in order to reduce the risk of false positive results from multiple tests.

In conclusion, our findings suggest that $\mathrm{QL}$ variables are important predictors of response to chemotherapy and survival in advanced breast cancer, and could be used to identify those patients who are least likely to benefit from chemotherapy. Specific hypotheses should be raised to test the predictive value of pain for survival, and dyspnoea for response to chemotherapy in women with advanced breast cancer. For clinicians it would also be important to identify the particular causes of pain and dyspnoea which influence prognosis.

\section{Acknowledgements}

This study was sponsored by Bristol-Myers Squibb International Corporation, Pharmaceutical Research Institute. J.A. Kramer was supported at the EORTC by the Lady Grierson Research Fellowship. 


\section{References}

1. Curran D, Sahmoud T, Therasse P, van Meerbeek J, Postmus PE, Giaccone G. Prognostic factors in patients with pleural mesothelioma: the European Organization for Research and Treatment of Cancer experience. J Clin Oncol 1998, 16, 145-152.

2. Simon R, Altman DG. Statistical aspects of prognostic factor studies in oncology. Br J Cancer 1994, 69, 979-985.

3. Coates A, Gebski V, Bishop JF, et al. for the Australian-New Zealand Breast Cancer Trials Group, Clinical Oncological Society of Australia. Improving the quality of life during chemotherapy for advanced breast cancer. A comparison of intermittent and continuous treatment strategies. N Engl J Med 1987, 317, $1490-1495$.

4. Coates A, Gebski V, Signorini D, et al. for the Australian New Zealand Breast Cancer Trials Group. Prognostic value of qualityof-life scores during chemotherapy for advanced breast cancer. $J$ Clin Oncol 1992, 10, 1833-1838.

5. Coates A, Thomson D, McLeod GRM, et al. Prognostic value of quality of life scores in a trial of chemotherapy with or without interferon in patients with metastatic malignant melanoma. Eur $J$ Cancer 1993, 29A, 1731-1734.

6. Seidman AD, Portenoy R, Yao T-J, et al. Quality of life in phase II trials: a study of methodology and predictive value in patients with advanced breast cancer treated with paclitaxel plus granulocyte colony-stimulating factor. J Natl Cancer Inst 1995, 87, 1316-1322.

7. Ganz PA, Lee JJ, Siau J. Quality of life assessment. An independent prognostic variable for survival in lung cancer. Cancer 1991, 67, 3131-3135.

8. Buccheri GF, Ferrigno D, Tamburini M, Brunelli C. The patient's perception of his own quality of life might have an adjunctive prognostic significance in lung cancer. Lung Cancer 1995, 12, 45-58.

9. Tamburini M, Brunelli C, Rosso S, Ventafridda V. Prognostic value of quality of life scores in terminal cancer patients. $J$ Pain Symptom Manage 1996, 11, 32-41.

10. Earlam S, Glover C, Fordy C, Burke D, Allen-Mersh TG. Relation between tumor size, quality of life, and survival in patients with colorectal liver metastases. J Clin Oncol 1996, 14, 171-175.

11. De Boer MF, Van den Borne B, Pruyn JF, et al. Psychosocial and physical correlates of survival and recurrence in patients with head and neck carcinoma: results of a 6-year longitudinal study. Cancer 1998, 83, 2567-2579.

12. Loprinzi CL, Laurie JA, Wieand HS, et al., for the North Central Cancer Treatment Group. Prospective evaluation of prognostic variables from patient-completed questionnaires. J Clin Oncol 1994, 12, 601-607.

13. Degner LF, Sloan JA. Symptom distress in newly diagnosed ambulatory cancer patients and as a predictor of survival in lung cancer. J Pain Symptom Manage 1995, 10, 423-431.

14. Aaronson NK, Ahmedzai S, Bergman B, et al. The European Organization for Research and Treatment of Cancer QLQ-C30: a quality of life instrument for use in international clinical trials in oncology. J Natl Cancer Inst 1993, 85, 365-376.

15. Coates A, Porzsolt F, Osoba D. Quality of life in oncology practice: prognostic value of EORTC QLQ-C30 scores in patients with advanced malignancy. Eur J Cancer 1997, 33, 1025-1030.
16. Dancey J, Zee B, Osoba D, et al. for the National Cancer Institute of Canada Clinical Trials Group. Quality of life scores: an independent prognostic variable in a general population of cancer patients receiving chemotherapy. Qual Life Res 1997, 6, $151-158$

17. Tannock IF, Osoba D, Stockler MR, et al. Chemotherapy with mitoxantrone plus prednisone or prednisone alone for symptomatic hormone-resistant prostate cancer: a Canadian randomized trial with palliative end points. J Clin Oncol 1996, 14, 1756-1764.

18. Sithisarankul P, Weaver VM, Diener-West M, Strickland PT. Multicollinearity may lead to artificial interaction: an example from a cross sectional study of biomarkers. Southeast Asian $J$ Trop Med Public Health 1997, 28, 404-409.

19. EORTC Breast Cancer Cooperative Group. Manual for Clinical Research in Breast Cancer. 3rd edn. The Netherlands, Excerpta Medica, 1998.

20. de Haes JCJM, van Knippenberg FCE, Neijt JP. Measuring psychological and physical distress in cancer patients: structure and application of the Rotterdam Symptom Checklist. $\mathrm{Br} J$ Cancer 1990, 62, 1034-1038.

21. Fayers P, Aaronson N, Bjordal K, Curran D, Groenvold M. EORTC QLQ-C30 Scoring Manual. 2nd edn. Brussels, EORTC Quality of Life Study Group, 1999.

22. Pocock SJ, Simon R. Sequential treatment assignment with balancing for prognostic factors in the controlled clinical trial. Biometrics 1975, 31, 103-115.

23. Kaplan EL, Meier P. Nonparametric estimation from incomplete observations. J Am Stat Assoc 1958, 53, 457-481.

24. Kenneth RH. Graphical methods for assessing violations of the proportional hazards assumption in Cox regression. Stat Med 1995, 14, 1707-1723.

25. SAS/STAT Software. Changes and Enhancements 6.11. Cary NC, USA, SAS Institute Inc, 1996.

26. Paridaens R, Biganzoli L, Bruning P, et al. Taxol versus Doxorubicin as first-line single agent chemotherapy for metastatic breast cancer - an EORTC randomized study with crossover. $J$ Clin Oncol 2000, 18, 724-733.

27. Williams MR, Todd JH, Nicholson RI, Elston CW, Blamey RW, Griffiths K. Survival patterns in hormone treated advanced breast cancer. Br J Surg 1986, 73, 752-755.

28. Robertson JFR, Dixon AR, Nicholson RI. Confirmation of a prognostic index for patients with metastatic breast cancer treated by endocrine therapy. Breast Cancer Research and Treatment 1992, 22, 221-227.

29. Blanco G, Holli K, Heikkinen M, Kallioniemi OP, Taskinen P. Prognostic factors in recurrent breast cancer: relationships to site of recurrence, disease-free interval, female sex steroid receptors, ploidy and histological malignancy grading. Br J Cancer 1990, 62, 142-146.

30. Herndon JE, Fleishman S, Kornblith AB, Kosty M, Green MR, Holland J. Is quality of life predictive of the survival of patients with advanced non-small cell lung carcinoma? Cancer 1999, 85, 333-340.

31. Ramirez AJ, Towlson KE, Leaning MS, Richards MA, Rubens RD. Do patients with advanced breast cancer benefit from chemotherapy? Br J Cancer 1998, 78, 1488-1494.

32. Hauck WW, Miike R. A proposal for examining and reporting stepwise regressions. Stat Med 1991, 10, 711-715. 